\title{
Effect of Maternal Protein Restriction in Rats on Cardiac Fibrosis and Capillarization in Adulthood
}

\author{
KYUNGJOON LIM, MONIKA A. ZIMANYI, AND M. JANE BLACK \\ Department of Anatomy and Cell Biology, Monash University, Clayton, Victoria 3800, Australia
}

\begin{abstract}
This study examines the effect of maternal protein restriction in rats on levels of cardiac fibrosis, myocardial capillarization, and media:lumen ratio of intramyocardial arteries in adult offspring. Female Wistar Kyoto rats were fed either a normal protein diet (NPD; 20\% casein) or a low-protein diet (LPD; $8.7 \%$ casein) during pregnancy and lactation. Female offspring (seven per group) were weaned at $4 \mathrm{wk}$ of age and grown to adulthood. At $24 \mathrm{wk}$ of age, the offspring were perfusion fixed. Cardiac fibrosis and media:lumen ratio of intramyocardial arterioles was assessed using image analysis and cardiac capillarization was stereologically investigated. Body weights at 2 and $24 \mathrm{wk}$ of age were significantly reduced (31\% and $8 \%$, respectively) in the LPD offspring; however, heart size was not different at 24 wk. Importantly by adulthood, there was a significant $15 \%$ increase in left ventricular interstitial fibrosis in LPD offspring. There were no differences in levels of perivascular fibrosis, myocardial capillarization, or in the media:lumen ratio of intramyocardial arteries between groups. Because cardiac fibrosis is associated with impaired cardiac contractility and arrhythmia, our results suggest that induction of interstitial fibrosis may contribute to the increased cardiac disease in adult subjects who were exposed to an adverse intrauterine environment. (Pediatr Res 60: 83-87, 2006)
\end{abstract}

$M^{a}$ any epidemiologic studies have shown that subjects exposed to perturbations in early development have an increased incidence of cardiovascular disease later in life (1-3). It is likely that an adverse intrauterine environment may permanently reduce the numbers of cells/functional units in vital organs, which in turn will affect postnatal organ function. Recent experimental studies of reduced nephron endowment in kidneys, as a result of maternal protein restriction, and subsequent detrimental effects on renal function later in life support this concept (4); whether this is the case in the heart has not yet been elucidated. Interestingly in a recent study in our laboratory, we found that maternal protein restriction throughout pregnancy in rats leads to a reduced heart size and a concomitant decrease in the number of cardiomyocytes (5). Because cardiomyocytes in general cease proliferating soon after birth, with postnatal growth of the heart predominantly due to cardiomyocyte hypertrophy (6), these findings could have important adverse implications on the structure and

Received October 25, 2005; accepted March 8, 2006.

Correspondence: M. Jane Black, Ph.D., Department of Anatomy \& Cell Biology, P.O. Box 13C, Monash University, Victoria 3800, Australia; e-mail: jane.black@med. monash.edu.au.

The findings of this study have resulted from a project supported by the National Health and Medical Research Council of Australia.

DOI: 10.1203/01.pdr.0000220361.08181.c3 function of the heart later in life. We postulate that a decrease in the total number of cardiomyocytes in neonatal hearts will limit the capacity for physiologic cardiac growth in adulthood. We propose that when cardiomyocytes reach their limits of hypertrophy, further enlargement of the heart will then occur through increased deposition of extracellular matrix leading to cardiac fibrosis.

Indeed, accumulation of extracellular matrix structural proteins in the heart adversely affects myocardial viscoelasticity $(7,8)$ with accumulation of fibrillar collagen leading to cardiac dysfunction (9-11). In addition, the isolation of cardiomyocyte groups by collagens can cause a reduction in gap junctions in cardiac muscle and lead to electrical load variations, thus triggering arrhythmias (12).

Ultimately how well the heart functions is reliant on the supply of oxygen and nutrients to the highly active cardiomyocytes. There is recent evidence suggesting that growth of the myocardial vasculature in the fetus may be affected by perturbations in utero (13). Under normal conditions, the rate of coronary vascularization during development is regulated by the magnitude of cardiac growth (14). However, hypoxia is a strong stimulus of capillary growth (15), and recent studies suggest that hypoxia in utero may augment the growth of the myocardial vasculature (13). Whether maternal protein restriction affects the vascularization of the heart is currently unknown.

Hence, the aims of the present study were to compare the levels of cardiac fibrosis, levels of capillarization, and the media:lumen ratio of intramyocardial arteries in the hearts of adult offspring that were exposed to either a normal maternal protein diet or to a reduced maternal protein diet.

We have previously shown using this model that the number of cardiomyocytes is significantly reduced at birth in offspring exposed to maternal protein restriction when compared with control offspring (5). In this study, a comprehensive analysis of fibrosis (interstitial, reparative, and perivascular) throughout the entire heart (right and left ventricles) was undertaken using image analysis. The right ventricle acts as an internal negative control for the specific effects of

Abbreviations: LPD, low-protein diet; $\mathbf{L V}+\mathbf{S}$, left ventricular wall and adjoining septum; NPD, normal protein diet; RV, right ventricular wall 
increased wall pressure that is present in the left ventricle. In addition, a detailed stereological study of the cardiac vasculature has been undertaken in the adult heart of offspring exposed to maternal protein restriction compared with control hearts.

\section{METHODS}

Animals and diet treatment. Twelve-week-old female and male Wistar Kyoto breeder rats were obtained from the Australian Resource Centre, Perth. The female rats were divided into two groups and fed either an NPD (containing 20\% casein) or a LPD (containing $8.7 \%$ casein) during pregnancy and for $2 \mathrm{wk}$ after birth. Initially, the rats were familiarized to the diets for 2 wk before mating. The diets were commercially available, semipurified diets (Glen Forrest Stockfeeders, Glen Forrest, Western Australia) (Table 1) (16). Diet intake was monitored daily. The breeder rats were housed individually and maintained at a constant temperature of $21^{\circ} \mathrm{C}$. Food and water were administered ad libitum. At birth, the litters were reduced to eight pups. To avoid the mothers becoming stressed, the offspring were not weighed until 2 wk of age. Body weight was measured weekly from weaning until 24 wk of age. Offspring (eight per group) used in this study were derived from six litters in the NPD group and five litters in the LPD group. After birth, the offspring were housed with their mother until weaning at $28 \mathrm{~d}$, at which time they were housed two to three rats per cage and kept until 24 wk of age. Only female offspring were used in this experiment. We chose to use females as we have previously shown significant reductions in total cardiomyocyte number in the hearts at birth of female offspring exposed to maternal protein restriction (5). Whether cardiomyocyte number is also reduced in male intrauterine growth restriction (IUGR) offspring at birth is unknown.

The animal experiments were approved by the Monash University, Biochemistry, Anatomy and Microbiology Animal Ethics Committee and treatment and care of the animals conformed with the Australian code of practice for the care and use of animals for scientific purposes.

Measurement of heart weight and ventricular wall volume. At $24 \mathrm{wk}$ of age, the rats were anesthetized (sodium pentobarbitone, $40 \mathrm{mg} / \mathrm{kg}$ intraperitoneally; Nembutal; Rhone Merieux, Australia) and perfusion fixed retrogradely via the abdominal aorta with $4 \%$ paraformaldehyde in $0.1 \mathrm{~mol} / \mathrm{L}$ phosphate buffer at a perfusion pressure of $140 \mathrm{~mm} \mathrm{Hg}$. Before fixation, heparin sodium was administered via the aortic catheter to prevent the blood from clotting, papaverine hydrochloride to maximally dilate the vasculature, and potassium chloride to arrest the heart in diastole. The hearts were excised, trimmed of fat and connective tissue and randomly assigned an experimental number so that the researcher was blinded to the experimental treatment group (eight in the NPD group and eight in the LPD group).

The atria were excised from the heart and then the ventricles (right and left) plus the intraventricular septum were weighed. The ventricles were sliced into 1-mm thick slices using a razor blade cutting device. It is to be noted that one of the hearts from the LPD offspring was markedly damaged in the initial slicing so it was excluded from the study.

The volume of the left ventricular wall and adjoining septum $(L V+S)$ and right ventricular wall $(\mathrm{RV})$ were then determined using the Cavalieri principle (17).

Assessment of cardiac fibrosis. Every second heart slice was embedded in paraffin, sectioned at $5 \mu \mathrm{m}$, and stained with a $0.001 \%$ Sirius red diluted in picric acid. It is to be noted that all sections were stained with the same freshly prepared batch of picrosirius red stain.

Interstitial/reparative fibrosis. The picrosirius-stained sections were viewed using an Olympus microscope (Olympus BH-2, Japan) and the image

Table 1. Composition of semipurified diets fed to dams during pregnancy

\begin{tabular}{lcl}
\hline Diet composition (\% by weight) & LPD & NPD \\
\hline Casein (acid) & 8.7 & 20 \\
Sucrose & 10 & 10 \\
Starch (total) & 64.41 & 53.11 \\
Cellulose & 5 & 5 \\
Safflower oil & 7 & 7 \\
Methionine & 0.14 & 0.14 \\
Minerals (AIN_93_G) & 3.5 & 3.5 \\
Vitamins (AIN_93_G) & 1 & 1 \\
Choline chloride 50\% wt/wt & 0.25 & 0.25 \\
\hline
\end{tabular}

projected onto a monitor screen with the aid of a CCD camera (Sony XC-77CE CCD, Japan) interfaced with a computer. Each sampled field of view was displayed on the monitor and the amount of red staining collagen area per total tissue area was measured using Microsoft Computer Imaging Device M4 (MCID) software (Version 3.0, Rev 1.4, 1997, Imaging Research Canada). All sections were analyzed under $\times 200$ magnification. A systematic uniform random sample of eight fields per section (six fields from the $\mathrm{LV}+\mathrm{S}$ and two fields from the RV) was undertaken to analyze the levels of interstitial/reparative fibrosis. Collagen area was determined by measuring the area of red-stained tissue within a given field and the relative proportion of interstitial/reparative fibrosis subsequently determined (18).

Perivascular fibrosis. For the study of perivascular fibrosis, each $\mathrm{LV}+\mathrm{S}$ section was initially scanned under $\times 20$ magnification and the number of myocardial arteries with a diameter ranging from about 150 to $400 \mu \mathrm{m}$ were counted and allocated a number. Two myocardial arteries were then selected for analysis, according to the method described by Nyengaard and Marcussen (19).

To determine the area of the lumen and adventitia of each artery, the outline of the lumen and the adventitia was traced under $\times 200$ magnification using image analysis. The cross-sectional area of the media of the same vessels was also measured. The areas of the lumen, media, and adventitia were subsequently calculated using Microsoft Computer Imaging Device M4 (MCID) software (Version 3.0, Rev 1.4, 1997, Imaging Research Canada)

The area of adventitia was taken as a measure of perivascular fibrosis. To account for differences in the size of arteries sampled, the adventitia:lumen ratio was calculated as a relative measure of perivascular fibrosis (20).

The area of the media was also measured and the media:lumen ratio for the myocardial arteries was also determined (20).

Sampling of the myocardium using the "Orientator." The remaining heart slices (approximately eight slices per heart) were used for the stereological analysis of capillarization in the heart. Due to the anisotropic nature of the myocardial capillaries, the systematic version 1 of the Orientator was used to generate isotropic sections of the myocardium (21).

The tissue was embedded in epon-araldite, with care taken to ensure that the cut surface according to the Orientator was flush with the top of the blocks. About eight sections from each block were cut at $1 \mu \mathrm{m}$ thickness and stained with toluidine blue.

Determination of Surface Area and Length of Myocardial Capillaries. One section from each slide was randomly chosen and projected on to the table top at a magnification of $\times 1475$ using an Olympus BX50F4 light microscope (Tokyo, Japan) modified with a projection arm. An orthogonal grid $(20 \times 20 \mathrm{~mm}$, nine-square grid) within an unbiased counting frame was superimposed on the projected image.

In each field of view, the number of grid points overlying myocardium $(P$ myo $)$ was counted and the number of capillary profiles $\left(N_{\text {cap }}\right)$, and the number of intersections of capillaries with horizontal and vertical grid lines $\left(I_{\mathrm{h}+\mathrm{v}}\right)$ were counted using the unbiased counting frame (22). Using a motorized stage, the fields of view were sampled in the $x$ and $y$ direction using uniform systematic random sampling. Ten fields of view per section (approximately 80 fields per heart) were analyzed.

The surface area density of myocardial capillaries, which is the surface area of capillaries per unit volume of $\mathrm{LV}+\mathrm{S}\left(S A_{\mathrm{V} \text { cap, myo }}\right)$ was calculated using the equation adapted from Mattfeldt et al. (21). The total surface area of capillaries in the $\mathrm{LV}+\mathrm{S}\left(S A_{\text {cap }, \mathrm{LV}+\mathrm{S}}\right)$ was then determined by multiplying the $S A_{\mathrm{V} \text { cap, myo }}$ by the volume of the $\mathrm{LV}+\mathrm{S}\left(V_{\mathrm{LV}+\mathrm{S}}\right)(21,23)$

The length density of myocardial capillaries, which is the length of capillaries per unit volume of $\mathrm{LV}+\mathrm{S}\left(L_{\mathrm{V} c a p}\right.$, myo $)$, was determined according to Mattfeldt et al. (21). The total length of capillaries in the $\mathrm{LV}+\mathrm{S}$ $\left(L_{\text {cap, } L V+S}\right)$ was determined by multiplying the length of myocardial capillaries per unit volume of the $\mathrm{LV}+\mathrm{S}\left(L_{\mathrm{Vcap}, \text { myo }}\right)$ by $V_{\mathrm{LV}+\mathrm{S}}(21,23)$.

Capillary diffusion radius was determined according to the formula:

$$
\text { Diffusion radius }=\sqrt{\frac{1}{\pi \times \text { Length density }}}
$$

Statistical analysis. Statistical analysis of the data was carried out using a computer-based statistical program, Graphpad Prism (version 3 for windows, Graphpad Software, San Diego, CA). To determine whether there were significant differences in the data between the two experimental groups at 24 wk of age, a two-tailed unpaired $t$ test was used. Changes in body weight over time were analyzed using two-way analysis of variance (ANOVA) with repeated measures. The results are expressed as means \pm standard error of the mean (SEM). Statistical significance was accepted at $p \leq 0.05$. 


\section{RESULTS}

Body weights. Maternal protein restriction during pregnancy and lactation led to marked effects on body weight of the offspring that persisted into adulthood (Fig. 1).

At 2 wk of age, the body weights of the LPD offspring were significantly reduced compared with the NPD offspring such that body weights were $31 \%$ lower in the LPD offspring when compared with the NPD controls (Table 2). Between 8 and 12 wk of age, there was a rapid increase in body growth in the LPD offspring with body weights surpassing those of controls at $8 \mathrm{wk}$ of age. At about $12 \mathrm{wk}$ of age, growth in the LPD offspring appeared to slow down relative to controls such that at $24 \mathrm{wk}$ of age, there was a significant (8\%) reduction in body weight in the LPD offspring compared with controls (Table 2).

Heart weight and ventricular wall volumes. At $24 \mathrm{wk}$ of age, there was no significant difference in heart weights or heart weight:body weight ratio in the LPD offspring compared with controls (Table 2).

There was also no significant difference in $\mathrm{LV}+\mathrm{S}$ wall volume and RV volume in the hearts of the NPD and LPD offspring (Table 2).

Interstitial/reparative fibrosis. There was no significant difference in the levels of interstitial fibrosis in the RV of the LPD and NPD offspring (4.64 $\pm 0.36 \%$ and $5.28 \pm 0.48 \%$ collagen volume fraction, respectively). Importantly, there was a $15 \%$ increase in the levels of interstitial fibrosis in the $\mathrm{LV}+\mathrm{S}$ of the LPD offspring compared with the NPD control (Fig. 2). During the systematic sampling of the $\mathrm{LV}+\mathrm{S}$, there were no apparent areas of reparative fibrosis (scarring) in hearts from either the LPD or NPD offspring.

Perivascular fibrosis. There was no indication of increased levels of perivascular fibrosis in the LPD offspring with no significant difference in the adventitia:lumen ratio of intramyocardial arteries in the LPD and NPD groups (Fig. 3A).

Media:lumen ratio of intramyocardial arteries. There was no significant difference in the media:lumen ratio of intramyocardial arteries in the LPD and NPD offspring (Fig. 3B).

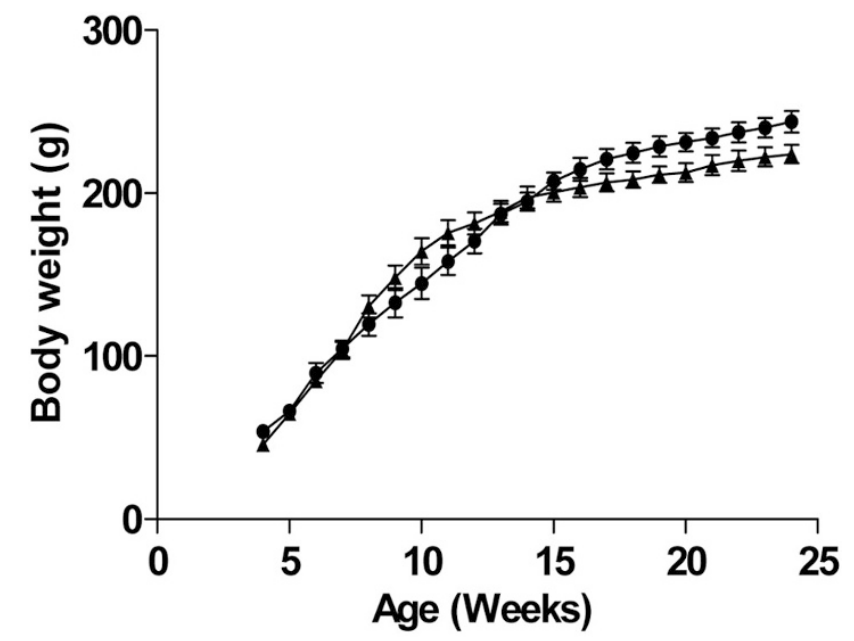

Figure 1. Body weights in LPD offspring $(\boldsymbol{\Delta})$ and NPD offspring $(\bullet)$ from the time of weaning ( $4 \mathrm{wk}$ of age) until adulthood (24 wk of age). Data were analyzed with a two-way ANOVA with factors: maternal diet (LPD vs NPD; $p=0.0219)$, age $(p<0.0001)$, and interaction $(p=0.0026)$.
Table 2. Body weights (at 2 and 24 wk of age), heart weights, heart weight:body weight ratios, $R V$ volumes, and $L V+S$ volumes of NPD $(n=8)$ and LPD offspring $(n=7)$ at 24 wk of age

\begin{tabular}{llc}
\hline & \multicolumn{1}{c}{ NPD } & LPD \\
\hline Body weight at 2 wk of age $(\mathrm{g})$ & $23.78 \pm 1.13$ & $16.43 \pm 0.46^{*}$ \\
Body weight at 24 wk of age $(\mathrm{g})$ & $243.8 \pm 6.7$ & $223.9 \pm 5.7 \dagger$ \\
Heart weight $(\mathrm{g})$ & $1.047 \pm 0.047$ & $0.972 \pm 0.026$ \\
Heart weight:body weight ratio & $4.30 \pm 0.01$ & $4.35 \pm 0.09$ \\
$\quad\left(\mathrm{mg} / \mathrm{g} \times 10^{3}\right)$ & & \\
Right ventricular wall volume $\left(\mathrm{mm}^{3}\right)$ & $430.0 \pm 14.2$ & $376.4 \pm 26.0$ \\
$\mathrm{LV}+\mathrm{S}$ volume $\left(\mathrm{mm}^{3}\right)$ & $967.5 \pm 64.0$ & $878.5 \pm 36.9$ \\
\hline
\end{tabular}

$* p<0.05 ; \dagger p<0.01$ compared to NPD offspring.

Capillarization in the left ventricle. Maternal protein restriction during pregnancy and lactation appeared to have no effect on capillarization in the adult heart of LPD offspring. There was no significant difference in either capillary length density or total capillary length of myocardial capillaries in the hearts of the LPD and NPD offspring at 24 wk of age (Table 3). Similarly, there was no significant difference in capillary surface area density or total capillary surface area of myocardial capillaries in the hearts of NPD and LPD offspring (Table 3). Diffusion radius of myocardial capillaries was not different between groups (Table 3).

\section{DISCUSSION}

In this study, offspring of rats exposed to maternal protein restriction during pregnancy and lactation showed no difference compared with controls in the level of capillarization, media:lumen ratio of intramyocardial arteries, or the levels of perivascular fibrosis in the heart by adulthood. However, there was a significant $(15 \%)$ increase in the level of interstitial fibrosis in the left ventricle. Hence, the findings of this study strongly suggest that exposure to maternal protein restriction early in life can lead to long-term adverse effects on the structural architecture of the heart. Because there was no difference in diffusion radius of myocardial capillaries or surface area and length densities of myocardial capillaries, the findings suggest that the blood supply to the myocardium was neither compromised nor augmented in the LPD offspring.

We predict that the increased deposition of fibrillar collagens observed in the present study is likely to be further exacerbated when the heart is challenged to undergo hypertrophy in adulthood, such as that seen with obesity or following induction of hypertension. In this regard, it was interesting to note that there was an acceleration in body growth of the LPD offspring during the period of adolescence (8-12 wk of age) when compared with control offspring. Body weight gain after this time was reduced compared with controls such that at 24 wk of age body weights were significantly less than those of controls. This effect on postnatal growth in female LPD offspring is different from what we have previously observed in male LPD offspring exposed also to maternal protein restriction during pregnancy and lactation. In the male offspring, there was no evidence of catch-up growth, with postnatal body weights remaining consistently lower compared with those of controls (16). Indeed, it may have been the 

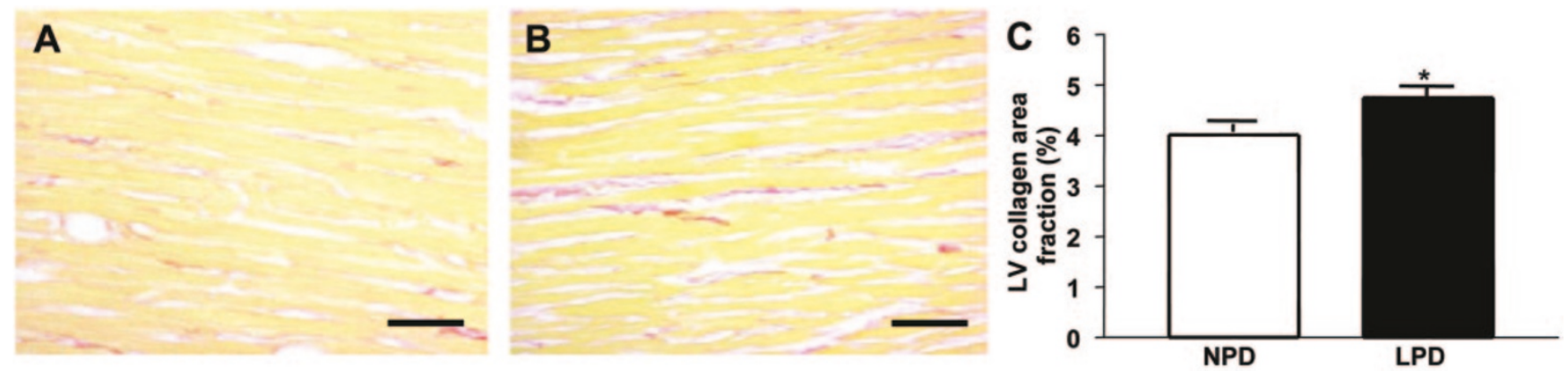

Figure 2. Representative picrosirius red-stained sections $(\times 200$ magnification; scale bar $=50 \mu \mathrm{m})$ showing interstitial collagen fibers (stained red) in the $\mathrm{LV}+\mathrm{S}$ of the heart from NPD offspring $(A)$ and LPD offspring $(B)$ and graph $(C)$ comparing the percentage of collagen area fraction in the LV $+\mathrm{S}$ of the NPD $(n=$ $8)$ and LPD $(n=7)$ offspring.
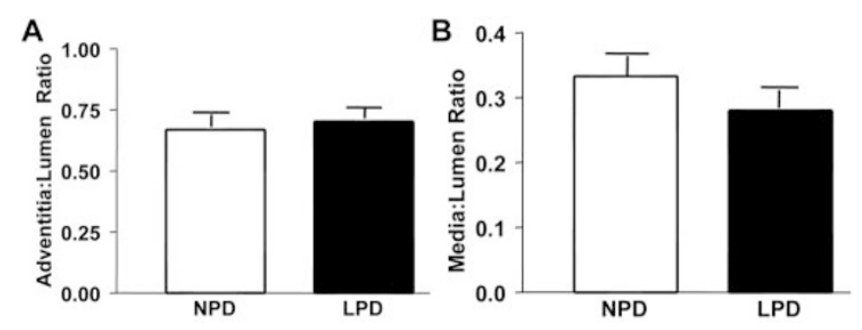

Figure 3. Adventitia:lumen ratio $(A)$ and media:lumen ratio $(B)$ of intramyocardial arteries in the LV $+\mathrm{S}$ of NPD $(n=8)$ and LPD $(n=7)$ offspring.

Table 3. Capillary length density, total capillary length, diffusion radius, capillary surface area density, and total capillary surface area of myocardial capillaries in the $L V+S$ of NPD $(n=8)$ and $L P D(n=7)$ offspring at 24 wk of age

\begin{tabular}{lcc}
\hline & NPD & LPD \\
\hline Capillary length density $\left(\mathrm{mm} / \mathrm{mm}^{3} \times 10^{3}\right)$ & $4.41 \pm 0.25$ & $4.78 \pm 0.36$ \\
Total capillary length $\left(\mathrm{mm} \times 10^{6}\right)$ & $4.30 \pm 0.35$ & $4.16 \pm 0.30$ \\
Diffusion radius $\left(\mathrm{mm} \times 10^{-3}\right)$ & $8.296 \pm 0.386$ & $8.560 \pm 0.228$ \\
Capillary surface area density $\left(\mathrm{mm}^{2} / \mathrm{mm}^{3}\right)$ & $67.69 \pm 4.60$ & $65.45 \pm 5.30$ \\
Total surface area $\left(\mathrm{mm}^{2} \times 10^{3}\right)$ & $64.05 \pm 2.99$ & $56.89 \pm 4.26$ \\
\hline
\end{tabular}

accelerated catch-up in body growth in our female LPD offspring that led to the induction of left ventricular interstitial fibrosis in these animals.

In this study, the increased levels of fibrosis in the cardiac interstitium in LPD offspring was specific to the left ventricle with no apparent increase in interstitial fibrosis in the right ventricle, suggesting that the fibrosis occurred in response to the hemodynamic load in the left ventricle. In this regard, a number of studies have reported that maternal protein restriction can lead to elevations in blood pressure in adulthood $(4,24,25)$. Although we have reported on a number of occasions that this is not the case in our model of maternal protein restriction during pregnancy and lactation in Wistar Kyoto rats $(16,26)$, these previous studies were all conducted using male offspring. We did not measure blood pressure in the female LPD offspring in the present study, nor did we measure blood pressure in female offspring in previous studies. Hence, we cannot rule out that there may have been an elevation in blood pressure in our female LPD offspring. Indeed, because the increase in interstitial fibrosis is specific to the $\mathrm{LV}+\mathrm{S}$, this would support the concept that the induction of fibrosis is secondary to an elevation in blood pressure. In future studies, we will address this issue and determine whether there are gender differences in blood pressure responses in our adult rat offspring exposed to maternal protein restriction.

In the present study, there was no evidence of increased perivascular fibrosis in the $\mathrm{LV}+\mathrm{S}$ of LPD offspring compared with controls, and there was no evidence of reparative fibrosis in either ventricle. Our findings are supported by the recent study by Briscoe et al. (27), where they showed increased accumulation of collagen in the interstitium of the left ventricle in adolescent guinea pigs that were growth restricted in utero, due to uterine artery ligation. Also in accordance with our findings they found no significant differences in the levels of perivascular fibrosis in the hearts of their adolescent growth-restricted offspring (27).

In general, the development of the vasculature in the fetal heart is proportional to cardiac mass, with heart development closely linked temporally and spatially to the regulated vascularization (14). However, there is some evidence to suggest that there is augmentation of myocardial vascular growth in the IUGR fetal heart exposed to hypoxia (13). Indeed, when hypoxia is present, angiogenesis is likely to be stimulated (15). In our study, capillarization of the heart in the LPD offspring was not different compared with the NPD offspring. Neither myocardial capillary surface area and length density nor total myocardial capillary surface area and length were different between the LPD and NPD offspring. Hence, there was no evidence of an augmented or decreased vasculature in the hearts of the LPD offspring in adulthood. In addition, we found that there was no difference in the diffusion radius of the capillaries in the heart, suggesting that blood supply to cardiomyocytes was not compromised in the adult hearts of the LPD offspring. However, it must be emphasized that we cannot assess from our study of rats in adulthood whether growth of the cardiac vasculature in the developing heart in utero was affected by maternal protein restriction.

In addition to effects on vascular growth, perturbations in utero have also been linked to postnatal vascular dysfunction (28-30). There appears to be regional heterogeneity in the extent of vascular dysfunction and also in the mechanisms involved that is endothelial, smooth muscle, or extracellular. Of particular relevance to the present study, maternal protein restriction has been shown to lead to blunted vasorelaxation in small resistance arteries $(31,32)$ Whether maternal protein 
restriction affects vascular function in the coronary vasculature is currently unknown.

In the present study, there was no evidence to suggest that there were structural increases in the smooth muscle content of the media of intramyocardial arteries in the heart of LPD offspring in adulthood, which, if evident, would imply increased contractility to vasoactive stimuli (33). The media:lumen ratios of the intramyocardial arteries were not different between the LPD and NPD offspring. Two recent studies have looked at the effect of perturbations in utero on the structure of the blood vessel wall in adulthood. Contrary to our findings in the coronary arteries, Briscoe et al. (27) reported an increased medial wall thickness, decreased luminal area, and an increase in medial elastic layers in the aorta of adolescent guinea pigs that were growth restricted in utero. Alternatively, in another rat study, IUGR due to placental insufficiency was associated with impaired vascular growth leading to a reduced lumen diameter, which persisted into adulthood (34). The differences in findings between studies demonstrate the complexity of the growth mechanisms in the blood vessel wall in vivo. The observed differences between our findings and those of other studies probably relate to differences in the site of the vessels sampled, species, or the model of in utero perturbation.

In conclusion, in this study, we have shown that maternal protein restriction during pregnancy and lactation does not affect the capillarization in the heart in adulthood, but importantly appears to lead to increased levels of interstitial fibrosis. Because cardiac fibrosis is associated with impaired cardiac contractility and arrhythmia, our results suggest that this may be a likely contributor to the increased cardiac disease in adult subjects that were exposed to an adverse intrauterine environment.

\section{REFERENCES}

1. Barker DJ, Osmond C, Golding J, Kuh D, Wadsworth ME 1989 Growth in utero, blood pressure in childhood and adult life, and mortality from cardiovascular disease. BMJ 298:564-567

2. Leon DA, Lithell HO, Vagero D, Koupilova I, Mohsen R, Berglund L, Lithell UB, McKeigue PM 1998 Reduced fetal growth rate and increased risk of death from ischaemic heart disease: cohort study of 15000 Swedish men and women born 1915-29. BMJ 317:241-245

3. Roseboom TJ, Van Der Meulen JH, Ravelli AC, Osmond C, Barker DJ, Bleker OP 2003 Perceived health of adults after prenatal exposure to the Dutch famine. Paediatr Perinat Epidemiol 17:391-397

4. Woods LL, Ingelfinger JR, Nyengaard JR, Rasch R 2001 Maternal protein restriction suppresses the newborn renin-angiotensin system and programs adult hypertension in rats. Pediatr Res 49:460-467

5. Corstius HB, Zimanyi MA, Maka N, Herath T, Thomas W, Van Der Laarse A, Wreford NG, Black MJ 2005 Effect of intrauterine growth restriction on the number of cardiomyocytes in rat hearts. Pediatr Res 57:796-800

6. Rudolph AM 2000 Myocardial growth before and after birth: clinical implications. Acta Paediatr 89:129-133

7. Bing OH, Matsushita S, Fanburg BL, Levine HJ 1971 Mechanical properties of rat cardiac muscle during experimental hypertrophy. Circ Res 28:234-245

8. Thiedemann KU, Holubarsch C, Medugorac I, Jacob R 1983 Connective tissue content and myocardial stiffness in pressure overload hypertrophy. A combined study of morphologic, morphometric, biochemical, and mechanical parameters. Basic Res Cardiol 78:140-155

9. Capasso JM, Palackal T, Olivetti G, Anversa P 1990 Severe myocardial dysfunction induced by ventricular remodelling in aging rat hearts. Am J Physiol 259:H1086H1096

10. Weber KT, Brilla CG, Janicki JS 1993 Myocardial fibrosis: functional significance and regulatory factors. Cardiovasc Res 27:341-348

11. Burlew BS, Weber KT 2002 Cardiac fibrosis as a cause of diastolic dysfunction Herz 27:92-98

12. Spach MS, Boineau JP 1997 Microfibrosis produces electrical load variations due to loss of side-to-side cell connections: a major mechanism of structural heart disease arrhythmias. Pacing Clin Electrophysiol 20:397-413

13. Davis L, Roullet JB, Thornburg KL, Shokry M, Hohimer AR, Giraud GD 2003 Augmentation of coronary conductance in adult sheep made anaemic during fetal life. J Physiol 547:53-59

14. Tomanek RJ, Hu N, Phan B, Clark EB 1999 Rate of coronary vascularization during embryonic chicken development is influenced by the rate of myocardial growth. Cardiovasc Res 41:663-671

15. Tomanek RJ, Lund DD, Yue X 2003 Hypoxic induction of myocardial vascularization during development. Adv Exp Med Biol 543:139-149

16. Zimanyi MA, Bertram JF, Black MJ 2004 Does a nephron deficit in rats predispose to salt-sensitive hypertension? Kidney Blood Press Res 27:239-247

17. Gundersen HJ, Bendtsen TF, Korbo L, Marcussen N, Moller A, Nielsen K, Nyengaard JR, Pakkenberg B, Sorensen FB, Vesterby A 1988 Some new, simple and efficient stereological methods and their use in pathological research and diagnosis. APMIS 96:379-394

18. Yu HC, Burrell LM, Black MJ, Wu LL, Dilley RJ, Cooper ME, Johnston CI 1998 Salt induces myocardial and renal fibrosis in normotensive and hypertensive rats. Circulation 98:2621-2628

19. Nyengaard JR, Marcussen N 1993 The number of glomerular capillaries estimated by an unbiased and efficient stereological method. J Microsc 171:27-37

20. Jones ES, Black MJ, Widdop RE 2004 Angiotensin AT2 receptor contributes to cardiovascular remodelling of aged rats during chronic AT1 receptor blockade. J Mol Cell Cardiol 37:1023-1030

21. Mattfeldt T, Mall G, Gharehbaghi H, Moller P 1990 Estimation of surface area and length with the orientator. J Microsc 159:301-317

22. Gundersen HJ 1978 Estimators of the number of objects per area unbiased by edge effects. Microsc Acta 81:107-117

23. Black MJ, Bertram JF, Johnston CI 2001 Effect of angiotensin-converting enzyme inhibition on myocardial vascularization in the adolescent and adult spontaneously hypertensive rat. J Hypertens 19:785-794

24. Langley SC, Jackson AA 1994 Increased systolic blood pressure in adult rats induced by fetal exposure to maternal low protein diets. Clin Sci 86:217-222

25. Vehaskari VM, Aviles DH, Manning J 2001 Prenatal programming of adult hypertension in the rat. Kidney Int 59:238-245

26. Zimanyi MA, Denton KM, Forbes JM, Thallas-Bonke V, Thomas MC, Poon F, Black MJ 2006 Developmental nephron deficit is associated with increased susceptibility to secondary renal injury due to advanced glycation end-products. Diabetologia 49:801-810

27. Briscoe TA, Rehn AE, Dieni S, Duncan RJ, Wlodek ME, Owens JA, Rees SM 2004 Cardiovascular and renal disease in the adolescent guinea pig after chronic placental insufficiency. Am J Obstet Gynecol 191:847-855

28. Leeson CP, Whincup PH, Cook DG, Mullen MJ, Donald AE, Seymour CA, Deanfield JE 1997 Flow-mediated dilation in 9- to 11- year old children: the influence of intrauterine and childhood factors. Circulation 96:2233-2238

29. Payne JA, Alexander BT, Khalil RA 2003 Reduced endothelial vascular relaxation in growth-restricted offspring of pregnant rats with reduced uterine perfusion. Hypertension 42:768-774

30. Roghair RD, Lamb FS, Bedell KA, Smith OM, Scholz TD, Segar JL 2004 Lategestation betamethasone enhances coronary artery responsiveness to angiotensin II in fetal sheep. Am J Physiol Regul Integr Comp Physiol 286:R80-R88

31. Brawley L, Itoh S, Torrens C, Barker A, Bertram C, Poston L, Hanson M 2003 Dietary protein restriction in pregnancy induces hypertensions and vascular defects in rat male offspring. Pediatr Res 54:83-90

32. Torrens C, Brawley L, Barker AC, Itoh S, Poston L, Hanson MA 2003 Maternal protein restriction in the rat impairs resistance artery but not conduit artery function in pregnant offspring. J Physiol 547:77-84

33. Mulvany MJ, Hansen OK, Aalkjaer C 1978 Direct evidence that the greate contractility of resistance vessels in spontaneously hypertensive rat is associated with a narrowed lumen, a thickened media, and an increased number of smooth muscle cell layers. Circ Res 43:854-864

34. Brodszki J, Lanne T, Marsal K, Ley D 2005 Impaired vascular growth in late adolescence after intrauterine growth restriction. Circulation 111:2623-2628 